\title{
¿Futuro limitado de la integración latinoamericana para impulsar el desarrollo?*
}

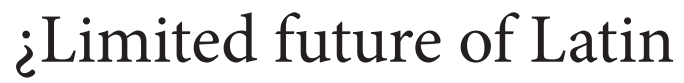 American integration to promote development?}

\author{
Ignacio Gómez Roldán** \\ Olga Marina García Norato***
}

Recibido: 2 de abril de 2014

Revisión: 23 de mayo de 2014

Aprobación: 11 de septiembre de 2014

\section{Resumen}

El fortalecimiento de los procesos de integración económica estimula el desarrollo de los mercados latinoamericanos, con lo que contribuye a la construcción de la política

* Artículo de revisión teórica que forma parte de la línea de investigación en Economía de las Organizaciones, adscrita al Grupo de Investigación en Economía y Humanismo del Centro de Investigación Louis Joseph Lebret de la Facultad de Economía de la Universidad Santo Tomás, sede Bogotá. Cómo citar este artículo: Gómez, I. \& García, O. (2014). ¿Futuro limitado de la integración latinoamericana para impulsar el desarrollo?. Revista CIFE, 16(24), 195- 215.

** Docente investigador de la Universidad Santo Tomás. Doctor en análisis económico de la UNED. Magíster en economía de la Universidad Nacional de Colombia. Especialista en evaluación social de proyectos de la Universidad de los Andes. Economista de la Universidad Javeriana. Coordinador de la línea de investigación en Economía de las Organizaciones. Correos electrónicos: ignacio.gomezr@usantotomas.edu.co; ignacio.gomez19@gmail.com

** Docente investigadora de la Universidad Santo Tomás. Doctora en historia, magíster en desarrollo rural y especialista en finanzas de la UPTC. Economista de la Universidad Santo Tomás, sede Bucaramanga. Líder del Grupo de Investigación en Economía y Humanismo, adscrito al Centro de Investigación Louis Joseph Lebret de la Facultad de Economía de la Universidad Santo Tomás, sede Bogotá. Correos electrónicos: olga.garcia@usantotomas.edu.co; olgarcia06@yahoo.es 
económica que fortalece la industrialización. El propósito del presente documento es analizar los criterios tenidos en cuenta para la industrialización de los países latinoamericanos y el papel de la integración latinoamericana con miras a identificar sus efectos reales en su desarrollo. La metodología consiste en una revisión bibliográfica que pone de relieve el papel y el contexto de los procesos de integración, y sus efectos esperados en las últimas décadas. Para ello, se revisan los siguientes aspectos: argumentos tradicionales de política para impulsar el desarrollo; integración económica como mecanismo de la política comercial favorecedora del progreso; cambios inesperados en el sector petrolero mundial; industrialización por sustitución de importaciones; revisión del mercado interno de las regiones; la des-industrialización al implantar ideas neoliberales, y el aumento de los acuerdos de libre comercio que profundizan la apertura y la globalización, con el propósito de ampliar los mercados, pero que estrangulan procesos de integración y vaticinan la muerte de la Comunidad Andina de Naciones (CAN).

Entre los hallazgos más relevantes de esta reflexión, está el papel secundario e irrelevante que ha cobrado el proceso de integración de la CAN, habida cuenta de que las negociaciones bilaterales (con Estados Unidos y la Unión Europea) y otros tratados condujeron a mayor vulnerabilidad de las subregiones. Los países latinoamericanos se desprotegieron ante los países que consideraban como amenazas sus procesos de industrialización y desarrollo. Esto favoreció el aumento de las importaciones de bienes industriales a la vez que se han dado procesos de desindustrialización.

Palabras clave: integración, desarrollo, Latinoamérica, exportaciones, sustitución de importaciones, industrialización.

Clasificación JEL: F02, F10, F13, F15, F43, O24, O25

\section{Abstract}

The strengthening of economic integration processes stimulates the development of Latin American markets, thus contributing to the construction of economic policy that strengthens industrialization. The purpose of this paper is to analyze the criteria considered for the industrialization of Latin American countries and the role of Latin American integration in order to identify the actual effects on their development. The methodology used in this document consists of a literature review that highlights the role and the context of integration processes and their expected effects in recent decades. To do this, the following aspects are reviewed: traditional policy arguments to promote development, economic integration as a mechanism of trade policy in favor of progress, unexpected changes in the global oil sector, import substitution industrialization, review 
of the regions' internal market, deindustrialization related to implemented neoliberal ideas and increased free trade agreements that deepen economic openness and globalization, in order to expand markets, but that strangle integration processes and predict the death of the Andean Community.

Among the most important findings of this reflection is the secondary and irrelevant role that has taken the process of integration of the Andean Community, given that bilateral negotiations (with the United States and the European Union) and other treaties led to increased vulnerability of the sub-regions. The Latin American countries were unprotected before countries considered to be threats to their industrialization and development. This led to an increase in industrial good imports while processes of deindustrialization have taken place.

Keywords: Integration, development, Latin America, exports, import substitution, industrialization

Classification JEL: F02, F10, F13, F15, F43, O24, O25 


\section{Introducción}

Las teorías tradicionales del comercio internacional fundamentadas en la oferta explican los beneficios del intercambio comercial entre los países, con la racionalidad de modelos de dos productos y dos países que tienen economías complementarias. En los años 90 fueron rescatadas para implantar políticas comerciales librecambistas, acordes con los intereses norteamericanos consignados en el Acuerdo de Washington y el Área de Libre Comercio de la Américas (ALCA). Sin embargo, frente a la búsqueda del desarrollo, buena parte de las limitaciones que enfrentan los países yace precisamente en una escasa oferta de productos de alto valor agregado, es decir que incluso si se considerara que el libre comercio arroja grandes beneficios para economías asimétricas, se deben reconocer las limitaciones de la oferta exportable, a fin de conseguir los beneficios que pregona la teoría.

Si bien la explicación del comercio exportador se asocia con adelantar caminos hacia el desarrollo, no es menos cierto que en diferentes tiempos, más que los problemas de la demanda, son las limitaciones de la oferta exportable (Bulmer-Thomas, 1998) las que dominan buena parte de las veces. Países en desarrollo han hecho grandes inversiones para poner en marcha proyectos productivos como enclaves del sector primario, que en la práctica conllevan el desaprovechamiento de las interrelaciones con el resto de la economía en el mejor uso alternativo de los recursos. La pérdida de oportunidades para incorporar mayor valor agregado en la producción retrasa el desarrollo, que pudiera impulsarse discrecionalmente desde posiciones conceptuales estratégicas de ventajas competitivas dinámicas.

Buena parte de los países latinoamericanos a finales del siglo XIX y comienzos del $\mathrm{XX}$ se han caracterizado por ser exportadores de productos primarios, jalonados por la dinámica globalizadora mundial. Con la depresión de los años 30 y el aprendizaje heredado de las guerras mundiales, las economías latinoamericanas entraron a cuestionar las bondades de las teorías tradicionales. En consecuencia, desde finales de los años 50, con las teorías de la dependencia, del intercambio desigual, del deterioro secular de los términos de intercambio, y con la influencia cepalina, estos países encontraron la necesidad de buscar alternativas de política económica que condujeran a la producción de bienes industriales como condición del crecimiento y el desarrollo de los pueblos.

En tal dirección, estas líneas recuperan, en primer lugar, argumentos tradicionales de política económica que han implementado los países hoy desarrollados y que les han servido para lograr procesos de industrialización. En segundo lugar, se expone cómo los procesos de integración se han planteado para estos propósitos desde ámbitos de cooperación y negociación, y desde la óptica de países de desarrollo relativo similar, en busca de la industrialización. En tercer lugar, se muestra cómo en la evolución de las 
interacciones-interdependencias mundiales y en las circunstancias de los shocks internacionales del petróleo, se generan los cambios y de ellos sacan provecho quienes tienen las posiciones más fuertes para imponer sus condiciones, con lo que afectan a los países en desarrollo.

Así, en la década de los 80 se dieron cambios que afectaron las políticas comerciales y los modos de pensar de aquello que se había planteado como la forma idónea de alcanzar el desarrollo; unos cambios hacia el tipo de políticas económicas propuestas por quienes tienen mayor desarrollo y condición hegemónica mundial. Así, fue mutando el concepto de integración aplicado por los países latinoamericanos, de manera tal que se pasó de la búsqueda de la industrialización por medio de un mercado interno regional a la búsqueda del crecimiento con propuestas librecambistas. En el último acápite del artículo, se expone que los países latinoamericanos intercambiaron la búsqueda de sus propios caminos hacia el desarrollo por recetas de teorías de libro no muy bien sustentadas en la realidad y que estrangulan los propios procesos de integración, considerados como un medio importante para conseguir la industrialización y el desarrollo.

De una previa concepción de inconveniencia de la intervención en los mercados, pregonada por la teoría clásica durante muchos años, se pasó desde la década de los 40 a una contextualización teórica general, que se impuso hasta los años 70, con la dominación de las teorías keynesianas en el manejo macroeconómico; se aceptaba entonces como práctica común la intervención del Estado en la economía; fueron también tiempos propicios para el montaje y el desarrollo del Estado de Bienestar en diferentes partes del mundo.

En América Latina, desde finales de los años 60, con diferencias entre los países y gran influencia de la Cepal, se fortalecieron las políticas de desarrollo hacia el interior. Se rescataron los planteamientos de Hamilton (1934) en los albores del siglo XVII, apoyando la intervención del Estado para favorecer la industrialización de Estados Unidos, y los de List (1856) en la industrialización alemana del siglo XIX. Por supuesto, los pensadores también se apoyaron en el británico John Stuart Mill, quien no obstante defender los principios de libertad a mediados del siglo XIX, propuso el argumento de la industria incipiente de la siguiente manera:

El único caso en que, aplicando solo los principios de la economía política, serían defendibles los derechos de protección aduanera, es cuando se aplican temporalmente (sobre todo cuando se trata de una nación joven y pujante) con la esperanza de dar carta de ciudadanía a una industria extranjera, que se adapta perfectamente a las condiciones del país. La ventaja de un país sobre otro en una rama de producción, a menudo deriva solo de haberla comenzado antes. Puede no haber una ventaja intrínseca de un país, o una desventaja de otro, sino solo una superioridad presente derivada de las habilidades y experiencias adquiridas. Un país que todavía no tiene esa capacidad y esa experiencia puede estar mejor dotado en 
otros aspectos para la producción que los que entraron primero en este campo; (...) además, no hay nada que refuerce más el mejoramiento en cualquier rama de la producción que el hecho de ensayarla en un nuevo conjunto de condiciones. Pero no puede esperarse que los individuos introduzcan una nueva manufactura por su propia cuenta (...) y soporten el peso de continuarla hasta que los productores adquieran un nivel de capacitación equivalente al de los productores tradicionales. Un derecho de aduana prolongado por un tiempo razonable puede, a veces, ser el modo menos inconveniente por el cual una nación puede aplicar un gravamen para apoyar un experimento. Pero es esencial que la protección se limite a casos que ofrezcan bastante seguridad de que la industria protegida podrá, después de un tiempo, sostenerse sola. (Mill, 1987, p. 922-923)

Aún con los viejos argumentos esgrimidos y llevados a la práctica por los países hoy desarrollados, se hicieron cuestionamientos sobre el derrame de los beneficios que se consiguen con mayor especialización y libre comercio como determinantes en la división internacional del trabajo (Krugman y Ofsfeld, 2001; Reinert, 2007; Winters y otros, 2004). Esto condujo a la implantación de modelos intervencionistas y al fortalecimiento de la regulación en los países en desarrollo.

Los diagnósticos realizados le apuntan a la conveniencia inaplazable de avanzar hacia estructuras productivas favorables hacia la industrialización, siendo coherentes con los desplazamientos poblacionales del campo hacia la ciudad. "De ahí, el significado fundamental de la industrialización de los países nuevos. No es ella un fin en sí misma sino el único medio de que disponen estos para ir captando una parte del progreso técnico y elevando progresivamente el nivel de vida de las masas" (Cepal, 1998).

De la revisión de la política comercial ejercida históricamente por los países que buscaban industrialización (en sus respectivos tiempos, Estados Unidos, Alemania, Japón, Italia, Australia, Rusia y España), el enfoque común fue el proteccionismo y no la liberalización comercial (Ramonet, 2000; Guerra-Borges, 2004). La "profunda transformación del Este asiático obedeció al papel clave que desempeñó un Estado desarrollista nacional aunado a una política industrial enérgica (impuesta tras una reforma agraria arrolladora) con miras a lograr el crecimiento y la competitividad internacional" (Kay, 1998). No es entonces de extrañar que América Latina haya elegido el camino de la protección y la intervención para fomentar la industrialización.

Esto no significaba dar la espalda a los sectores primarios, sino aprovechar y articular el potencial de los recursos de los países hacia la reforma de las estructuras productivas que evolucionaran hacia una mayor participación de las manufacturas, en las dinámicas generadoras de riqueza de los países. De allí que las políticas de sustitución de importaciones fueron articuladas con promoción y diversificación de exportaciones, de manera que al ampliar los mercados dentro de los procesos de integración, se impulsara el crecimiento y el desarrollo. 


\section{La integración económica como mecanismo de la política comercial favorecedora de la industrialización}

Los procesos de integración son complementarios o van de la mano de la globalización vinculados a proyectos políticos, lo que permite coordinación o definición de objetivos comunes de áreas mayores a los países mismos (Pipitone, 1996; Morales-Fajardo, 2007). Para impulsar el crecimiento del aparato industrial de las economías latinoamericanas que propendían a altos grados de intervención y proteccionismo, a partir de la segunda mitad del siglo XX se fortalecieron los procesos de integración regional en los marcos del Acuerdo General de Tarifas y Aranceles (GATT). Fue así que en América Latina nacieron, entre otros: el ALALG (que evolucionó posteriormente para convertirse en la ALADI), con acuerdos de alcance parcial, preferencias arancelarias regionales y área de preferencias económicas; y el Pacto Andino (que evolucionó posteriormente hacia la Comunidad Andina de Naciones - CAN), inicialmente con pretensiones de unión aduanera y luego de mercado común. Nacieron también los acuerdos centroamericanos, el NAFTA, el Mercado Común del Sur (Mercosur) y otros acuerdos. Entrado el nuevo milenio, siguieron aumentando los tratados de libre comercio (TLG) entre países geográficamente más lejanos.

En esta dirección, los acuerdos de integración incluían herramientas de política industrial como los Programas Sectoriales de Desarrollo Industrial -en el marco del Pacto Andino-, y a nivel nacional los programas de desagregación tecnológica, como tenía Colombia en cabeza de Colciencias. En el Pacto Andino, la posición era la protección frente a los países desarrollados, aunque no solo en lo comercial, y de allí las restricciones a la inversión extranjera que estaban reguladas por la decisión 24 del Acuerdo de Cartagena.

Era pues manifiesto, desde diferentes ámbitos como el financiero (líneas de crédito e instituciones especializadas), el interés nacional de los países en el desarrollo y la implementación de políticas sectoriales. Estas, por supuesto, contaban con estructuras arancelarias que favorecían la protección al valor agregado nacional y se aplicaban mayores tarifas a los productos industriales (con potencial producción o producción existente); tarifas intermedias para materias primas e insumos, y tarifas más bajas a bienes primarios y no producidos nacionalmente (Gómez, Moreira \& Santamaría, 1994).

En los años 60 y 70, se crearon organizaciones promotoras de las exportaciones de los países, que incluso hoy, modernizadas, apoyan su crecimiento y diversificación. En esta misma dirección, los mecanismos de importación-exportación se convirtieron en importantes instrumentos de apoyo al sector exportador, con la exoneración parcial o total de aranceles a la importación de las materias primas y los componentes que efectivamente 
se incorporaran en los productos de exportación. Así las instituciones y las políticas comerciales de los países latinoamericanos se orientaban hacia la promoción y el desarrollo de exportaciones de bienes industriales.

\section{Los cambios inesperados en el sector petrolero mundial desestabilizaron el ideario exportador latinoamericano}

En medio de estos procesos y cambios, se desataron los reclamos de los países de Oriente sobre la propiedad de los recursos petroleros que estaban en manos de las multinacionales originarias de los países occidentales. Fue así que entre los impactos provocados por las alzas en el precio del petróleo y la canalización de estos recursos financieros hacia la banca occidental, se encuentra el desmedido endeudamiento de los países latinoamericanos, debido al relajamiento de las condiciones crediticias que dio la banca internacional.

Esto dio como resultado la crisis de la deuda de muchos países de la región; crisis conocida en adelante como la "década perdida" y que significó, para muchos países, no solamente disminuciones importantes en el PIB, el empleo y la quiebra de muchas empresas, sino también el establecimiento de niveles inflacionarios no vistos antes. La intervención del Fondo Monetario Internacional (FMI) y el del Banco Mundial (BM), junto con el reclamo mismo de la banca privada internacional sobre las acreencias, se acompañó con planes de emergencia y rescate como el Plan Brady, que condicionó los créditos a los ajustes de políticas y metas sugeridas por el FMI.

Las lecturas de las experiencias exitosas de la industrialización asiática, fundamentadas en las exportaciones, impulsaron los diagnósticos que favorecían recomendaciones de política económica más afines con el pensamiento austríaco y la Escuela Económica de Chicago, favorecedoras de la liberalización y la desregulación de las economías.

En estos contextos de crisis de los países, surgió la propuesta de creación del Área de Libre Comercio para las Américas (ALCA) y del Consenso de Washington. Los estudios de Sachs y Warner (1995), y de Dollar y Kraay (2001) -que tuvieron gran divulgación-, invitaron con indicadores de gran sencillez, o quizás simpleza (Gómez, 2013), a la conveniencia de liberalizar las economías so pena de quedar sumidos en la pobreza y el subdesarrollo.

El ALCA se entiende a partir de las propuestas aperturistas que profundizan las reformas institucionales y la desregulación de los mercados, con la flexibilización del mercado laboral y la disminución del tamaño del Estado (Ocampo, 2001). De otro lado, se pronosticaban como efectos la liberalización y desgravación de las economías, y la desindustrialización y des-agrarización de las economías con tercerización temprana y 
perversa, en la cual la captura de rentas desplazaría la producción. En esta posición, se anticipaba ya mayor empobrecimiento, aumento de exclusión social, "especialización perversa", y explotación más intensiva de los recursos naturales (Garay, 2002; Pulecio, 2002).

En medio de la década perdida latinoamericana, comenzó a desarrollarse la Ronda de Uruguay, que daba señales de no concluir, dejando en incertidumbre a los países en los marcos regulatorios de las relaciones económicas internacionales promulgadas por el GATT. Con el interés de la ampliación de los mercados, proliferó la firma de nuevos acuerdos de integración en todo el mundo, los cuales incorporaron progresivamente más temas (como los servicios y la propiedad intelectual, de gran interés especialmente para los países desarrollados) en las negociaciones, con las desgravaciones arancelarias como apenas un tema más tras haber sido en el pasado el core de lo firmado.

\section{De la industrialización por sustitución de importaciones y un mercado interno de las regiones, a la des-industrialización al implantar las ideas neoliberales}

El enfoque de industrialización por sustitución de importaciones (ISI) buscaba crear condiciones de expansión de la demanda agregada, con crecimiento de los mercados internos. La política fiscal y monetaria, a partir de la intervención estatal de los gobiernos latinoamericanos, propendían a la contribución de la demanda agregada al desarrollo. No obstante, en la práctica, se preservó la estructura agraria, la permanencia de sus rentas y la exclusión de la población no relacionada con el sector moderno de la economía, de allí que se generaron fuerzas a favor y en contra de la industrialización (Misas, 2002).

Es preciso admitir que buena parte de las industrias no produjeron condiciones tecnológicas y administrativas suficientemente competitivas:

...la acumulación de capital no desembocó en la generalización de la relación salarial, e impidió la creación de un círculo virtuoso, a lo Veerduurn-Kaldor, de mayores salarios reales-mayor demanda efectiva - mayor nivel de producto-mayor nivel de productividad-, típicos de los modelos fordistas de acumulación. El limitado crecimiento de la demanda interior por bienes manufacturados, dio lugar a que el modelo de industrialización sustitutiva de importaciones llegara a su fin. (Misas, 2002, p. 295)

Se trató de complementar la industrialización por sustitución de importaciones, por medio de acuerdos subregionales que posibilitaban el crecimiento del sector externo de cada país; hacia dentro de la subregión se eliminaban las barreras al comercio, desistiendo de o aumentando la protección a terceros países, y entraron a jugar un papel 
importante la planeación y el direccionamiento o la intervención de los Estados. De allí que surgieron organizaciones supraestatales a nivel regional que tuvieron referentes en el GATT y en la experiencia integracionista de los europeos (Banco Interamericano de Desarrollo [BID], 2002, p. 36).

El no cumplimiento de expectativas bajo las políticas de la ISI, la crisis de la deuda de los años 80 y el fortalecimiento de las ideas neoclásicas, fueron el piso para las posteriores reformas de política comercial, y la redefinición del papel de los Estados en la economía y en un nuevo tipo de integración. La búsqueda de la reconversión industrial privilegió un mayor papel de la libertad de los mercados en la asignación de los recursos, en estas condiciones, bajo el pretexto de las oportunidades que ofrecía la globalización (Ocampo, 2001), el optimismo dado y el reconocimiento a la confianza que se había dado a la industrialización por sustitución de importaciones. Al persistir una gran dependencia de productos primarios (Ffrench-Davis, Muñoz \& Palma, 1998), las reformas aperturistas desplegaron hacia una mayor liberalización de importaciones, desregulación, privatizaciones, flexibilización del mercado laboral, descentralización, independencia del banco central, y búsqueda de un mayor equilibrio fiscal.

Los gestores de la apertura, basados en un diagnóstico superficial e incompleto, creían que la eliminación de barreras que limitaban la competencia externa era suficiente para inducir a las empresas a cambiar sus normas de producción y a buscar en los mercados externos las condiciones que les permitieran aprovechar plenamente las economías de escala. Sin embargo, los actores económicos, obrando racionalmente, reaccionaron en forma diferente de lo previsto por los partidarios de la apertura (...) Se dio lugar a una desaceleración en el ritmo de acumulación de capital en los sectores productores de bienes transables, a una pérdida del dominio sobre el mercado interno (...) La ausencia de un verdadero debate impidió precisar las ventajas y limitaciones de las reformas propuestas (...) Mediante una hábil combinación de los intereses organizados un activo vector de comunicación establecido (...) impusieron una versión dogmática amparados en su "saber" acerca de lo que era "correcto" y lo que era "incorrecto" en el debate sobre globalización e internacionalización de la economía (Misas, 2002, p. 295).

Las actuaciones imprudentes del desmantelamiento arancelario en América Latina no suavizaron el impacto de los cambios en las firmas; y una cantidad considerable de empresas fueron a la quiebra y la región sintió la pérdida de un número importante de puestos de trabajo. Es preciso anotar que en los casos de Corea, Taiwán y Japón, las actuaciones de liberalización fueron diferentes (Stiglitz, 2002). El énfasis de estos países se puso en políticas industriales y en las exportaciones manufactureras, y se guardó moderación y selectividad en la liberalización de importaciones, con miras a la preservación y expansión de la industria y a la consecución del desarrollo.

Así, mientras que la apertura de los países asiáticos se vinculó con estrategias de desarrollo, la apertura latinoamericana "se subordinó a una ideología" (Guerra-Borges, 2004, 
p. 110). Joseph Love (1994, p. 395), refiriéndose al proceso pragmático de industrialización suramericana, consignó que: "La industrialización de América Latina fue un hecho antes de que fuera una política, y una política antes de que fuera una teoría"; con ello, da a entender la existencia de falencias en la concepción y la eficacia de la implantación de las políticas emprendidas por los países suramericanos.

Los desequilibrios externos provocados por los menores precios de las materias primas impulsaron la implantación de modelos mixtos de desarrollo que combinaron la sustitución de importaciones, el crecimiento y la diversificación de las exportaciones, apoyados en la ampliación de los mercados, con los procesos de integración regional. De esta manera, lo que se alcanzaba con la sustitución de importaciones se lanzaba al mundo con estrategias de promoción de exportaciones, y con el direccionamiento de las políticas cambiarias (French-Davis, Muñoz y Palma, 1998; Ocampo, 2004 y 2008) y financieras, de las que se esperaba que contribuyeran al cambio social y no solo a la producción de manufacturas (Hirschman, 1971), de manera que se pudiera conseguir mayor estabilidad externa.

A partir de la experiencia de diferentes países desarrollados, se puede afirmar que:

...la apertura puede ser una oportunidad si se acompaña de una estrategia industrial. Gracias al apoyo de un Estado estratega, una economía abierta no es una economía "regalada" a los intereses exteriores, como cuando se trata solo de libre comercio. Una apertura controlada permite transformar el tejido industrial y preparar el país para las mutaciones necesarias. Pero no es fácil cambiar de una política pública a otra, debido a lo fuertes que pueden ser los conflictos de interés de los grupos económicos y sociales. Los grupos de presión influyen sobre la política económica y el interés de estos puede predominar sobre el interés general. (Salama, 2012, p. 44)

El sistema de las ideas neoliberales, formalmente coherente en abstracto, ha puesto de manifiesto su inadecuación a la realidad, porque la linealidad de su discurso a socie $\neg$ dades que en razón de su historia son disímiles y fracasan en su intento de ajustarlas a un esquema único. Quedó de manifiesto que el neoliberalismo se ha dirigido a sociedades que no existen. Y las que realmente existen, dolorosamente desengañadas, quieren ahora emprender de nuevo el camino de las búsquedas. Seguir de nuevo el rastro de su sinuoso destino. (GuerraBorges, 2004, p. 113)

Sin embargo, si las orientaciones de integración económica de tiempo atrás pretendían disminuir las vulnerabilidades de América Latina a las variaciones del sector externo, las reformas aperturistas aumentaron su exposición a los riesgos de shocks externos. Y si bien América Latina fue el conjunto de países que mejor siguió las instrucciones de acuerdo con las propuestas de la ortodoxia y de las ideas neoliberales, no es menos cierto que son muchos los países que han desandado caminos hacia el desarrollo tras darse procesos de pérdidas de rentabilidad y competitividad (Frenkel y Rapetti, 2011) concomitantes a procesos de desindustrialización (Echavarría y Villamizar, 2006; Ocampo 
y Martínez, 2011), que Pierre Salama (2012) califica de prematuros durante los últimos lustros.

Y es que la desindustrialización no solo se vincula con la reducción relativa y absoluta de los empleos industriales, sino también con la destrucción de una parte de su tejido industrial. Se habla entonces de la desindustrialización prematura de muchos países de América Latina (Salama, 2012), calificativo que podría acompañar al tipo de tercerización de las economías latinoamericanas, la cual se caracteriza por bajo valor agregado y por la afluencia de capitales especulativos.

\section{Aumentan los acuerdos de libre comercio que profundizan la apertura y la globalización con el propósito de ampliar los mercados y estrangulan los procesos de integración que vaticinan la muerte de la CAN}

Del viejo debate entre el desempeño económico y la política comercial, es preciso recordar que de acuerdo con los economistas liberales, el mayor libre comercio conduce a mayores tasas de crecimiento, mientras que otros argumentan que el proteccionismo ayuda al desempeño (Eduards, 1998). Desde la década de los 80, los países latinoamericanos ofrecieron mayor acceso a su mercado bajo acuerdos de integración, motivados por las experiencias asiáticas de apertura exportadora de manufacturas, y posteriormente por la perestroika, el derrumbamiento del muro de Berlín, la entrada de China al "capitalismo" y las políticas de Estados Unidos (que fortalecieron las ideas neoliberales).

Y es que bajo los acuerdos de integración se rompe, como excepción, la cláusula de la nación más favorecida (capítulo 34 del GATT-OMC), al ser considerados por la teoría como un second best (Riveiro, 2005; Tamames, 2010). Ante la mejor opción de completa libertad de comercio entre todos los países que propone la teoría tradicional y la frecuente protección que tienen los países, los acuerdos de integración tienden hacia el libre comercio entre sus participantes. Así, es preferible liberalizar el comercio entre pocos países que guardar una posición proteccionista frente a todos los países.

Las liberalizaciones unilaterales de algunos países latinoamericanos, que perseguían la eliminación del sesgo anti-exportador, siguieron entonces procesos crecientes de mayor liberalización valiéndose de una ola creciente de tratados de libre comercio bilaterales de tercera o cuarta generación. De manera tal que no obstante los países en desarrollo se han integrado más en la economía mundial durante las últimas décadas, la integración sigue siendo sesgada, llena de sorpresas y no ha tenido los beneficios esperados (Rodrik \& Rosenzweig, 2009; Riveiro, 2005). 
Entre los escépticos sobre los grandes beneficios que aporta la liberalización del comercio, están Paul Krugman (1994) y Dani Rodrik (1995), quienes han argumentado que el efecto de la apertura en el crecimiento, en el mejor de los casos, es muy tenue, y en el peor, es dudoso (Eduards, 1998). Y es que en los acuerdos de libre comercio entre países asimétricos se liberaliza el comercio y los beneficios no se reparten igual entre los participantes.

Romer (1986) y Lucas (1988) defienden la posición aperturista por sus efectos positivos en el crecimiento. Con otro matiz, Romer (1992), Grossman y Helpman (1991), y Barro y Sala-i-Martin (1995) señalan que los países más abiertos absorben mejor los avances tecnológicos. Barro y Sala-i-Martin (1995) consideran que en el caso de dos países, uno avanzado-innovador y otro pobre-imitador, la mayor tasa de equilibrio del crecimiento del país pobre dependerá de los menores costos de imitación y de su nivel de conocimientos (Eduards, 1998). Así, los países con más apertura gozan de mayores competencias para percibir y desarrollar las nuevas ideas (Obstfeld y Rogoff, 2000), y logran mayores crecimientos de la productividad y mayores apropiaciones de beneficios.

$\mathrm{Al}$ hacer las lecturas de las bondades del libre comercio, debe reconocerse la dificultad técnica y práctica de las investigaciones existentes para estimar indicadores de la orientación de la política comercial. Los estudios tienen fuertes limitaciones en los resultados entre la productividad total de los factores de la apertura y la tasa de crecimiento (Rodrik, 1995).

Si bien desde mediados de los años 50 Jean Timbergen (1954) identificaba la integración con el libre comercio en el mundo para los bienes agropecuarios e industriales, Bella Balassa (citado por Conesa, 1982) la concibió como un proceso en el cual varios países forman un grupo regional. Los procesos de integración se entienden como agrupaciones de países, y también, en el caso que nos ocupa y en contexto, su objetivo es "mejorar la presencia de América Latina en el escenario mundial y contrarrestar la influencia de Estados Unidos" (Quitral, 2009).

La integración es "un elemento central de las políticas de desarrollo, y en particular, un medio fundamental para acelerar el ritmo de crecimiento de las economías latinoamericanas" (Peñaranda, 1984). La integración, al conducir hacia la mejor asignación de los recursos en los procesos de sustitución de importaciones y promoción de exportaciones en la subregión (especialmente de manufacturas consideradas como motor de la economía), persigue entonces la eficiencia y la interdependencia para que la región obtenga autonomía económica.

De esta manera, los procesos de integración latinoamericana se entienden, especialmente hasta los años 80, en un contexto de desarrollo regional hacia dentro, con crecimiento 
de la producción y exportación industrial en las subregiones, e identifica a los países desarrollados (Estados Unidos y los países europeos, sobre todo) como amenazas o agentes activos que han contribuido al atraso de la región.

Llegados los años de la promulgación del ALCA y del Consenso de Washington, se entró en la era de un regionalismo abierto cuyo discurso se enmarca con la era de la globalización. En esta dirección, en los acuerdos de integración más relevantes entre países latinoamericanos, el Mercosur y la CAN, se observan procesos diferentes. Brasil sigue su propio camino diseñando su propia estrategia, y buscando por motivos políticos una integración que se concreta principalmente con Argentina y otros países de menor importancia en el Mercosur, cuya principal mirada no se encuentra en Estados Unidos.

El grupo Andino, buscando reestructurarse, se convirtió en la Comunidad Andina de Naciones, que luego de la salida de Venezuela y de los tratados de libre comercio bilaterales de Perú y Colombia con Estados Unidos, perforan el AEC en forma sustancial. Las inclinaciones políticas de Ecuador y Bolivia afines con Venezuela generan unas posiciones que favorecen el estrangulamiento de la CAN, especialmente al tener en cuenta que estos países siempre reclamaron su posición de menor desarrollo relativo para dilatar sus compromisos con la subregión.

En estas condiciones, la potencialidad del aprovechamiento de las subregiones para el desarrollo industrial se perdió. Adicionalmente, Perú y Colombia firmaron, cada uno por separado, tratados bilaterales con la Unión Europea.

En la memoria de los países latinoamericanos que recibieron la propuesta del ALCA, estaba presente la respuesta inmediata de la unión de los países para que se hiciera contrapeso en las negociaciones asimétricas con Estados Unidos. Pero muchos de los factores determinantes en el bajo alcance y en la duración real de los acuerdos de integración entre los países latinoamericanos se hicieron presentes para que las uniones no se dieran. Cada pequeño país latinoamericano terminó haciendo negociaciones "entre iguales" con Estados Unidos. De este modo, se perdió en los hechos una vieja idea expresada por Alfredo Guerra-Borges (2004, p. 110): "Solo una creciente cooperación latinoamericana podrá dar pie para una mejor defensa de los intereses de nuestros países frente a un mundo que es hostil, no tanto porque siempre agreda, sino porque en ningún momento abandona sus propósitos de subordinación"; una afirmación que tiene mucho sentido y validez ante los escenarios planteados.

Las condiciones de los países en el transcurrir del tiempo no son repetibles, y el desaprovechamiento de determinadas circunstancias hace que se pierdan oportunidades históricas para el manejo de ciertos instrumentos de política comercial que han dado resultados en otros países. 
Las fuerzas de la globalización han reducido aún más las posibilidades de maniobrar a través de políticas nacionales de desarrollo en comparación con el periodo de ISI, confirmándose así uno de los principios claves de la teoría de la dependencia. El poder de los mercados internacionales rige con más fuerza todavía que en el pasado y los Estados deben tomar en cuenta estas presiones de los mercados globales. (Kay, 1998, p. 7)

Es previsible entonces, como se ha visto en los últimos lustros, que se siga presentando una mayor desindustrialización de los países latinoamericanos con una tercerización precaria, ante la adopción de políticas de mayores libertades de los mercados, las privatizaciones, la menor intervención de los Estados, y una mayor expansión de la globalización con las grandes corporaciones.

\section{A manera de conclusión}

Los acuerdos de integración propendían a un alto nivel de proteccionismo hacia terceros países (especialmente frente a los países desarrollados) y a una menor protección hacia el interior de la subregión. De esta manera, se buscaba la ampliación de los mercados de cada país con los miembros de las respectivas subregiones. En estas condiciones, se protegía a la naciente o incipiente industria nacional, de las importaciones provenientes de los países desarrollados y se impulsaban las exportaciones entre los países miembros de las subregiones.

Las crisis petroleras cuyos efectos se observaron en todo el globo dieron lugar a nuevos planteamientos paradigmáticos con la aparición de fenómenos como la estanflación y las lecturas sobre el agotamiento del modelo de sustitución de importaciones, en el caso latinoamericano.

Las economías desarrolladas usualmente pasan por procesos de desindustrialización a partir de cierto ingreso per cápita, mientras que los países emergentes latinoamericanos tienden hacia la desindustrialización antes de que su ingreso per cápita llegue al nivel de las naciones desarrolladas a comienzos del proceso.

Sería un error afirmar que todas las aperturas per se conducen a la desindustrialización. Es importante tener en cuenta cuándo, cómo, dónde, con quiénes se hace, y sobre todo, su relación con las intervenciones del Estado en las políticas macroeconómicas y sectoriales.

Es decir que los países de la CAN terminaron en el juego de las naciones desarrolladas con liberalización de sus economías en negociaciones totalmente asimétricas, de las que por mucho tiempo buscaron protegerse. Tratados de libre comercio de cuarta generación, con la promesa de acceso a mercados, para los cuales no se dispone de una oferta exportable consolidada. 


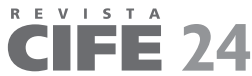

ISSN: 0124-3551 / Año 16, No 24 / enero-junio / pp. 195-215

La complejidad de la política comercial, al combinar diferentes variables como aranceles, cuotas, licencias, prohibición de importaciones, control de cambios y acuerdos de integración, franjas de precios, cláusulas de salvaguardias, subsidios (que combinan estos y otros instrumentos), entre otros, muestra las dificultades para estudiar el fenómeno. Esta política debe sincronizarse con la política y la estrategia de industrialización en un contexto de globalización y regionalización. 


\section{Referencias}

Banco Interamericano de Desarrollo - BID. (2002). Más allá de las fronteras. El nuevo regionalismo en América Latina. Washington: Autor.

Barro, R.J. and X. Sala-i-Martin, 1995, Economic growth, McGraw Hill, Boston, MA.

Bulmer-Thomas, V. (1998). La historia económica de América Latina desde la independencia. México, D.F.: Fondo de Cultura Económica.

Casilda Béjar, R. (2004). América Latina y el Consenso de Washington. Boletín Económico de ICE n. ${ }^{\circ}$ 2803, 19-38. Recuperado de http://biblioteca.hegoa.ehu.es/system/ ebooks/14120/original/America_Latina_y_el_consenso_de_Washington.pdf

Comisión Económica para América Latina y el Caribe - Cepal. (2002). Globalización y desarrollo. Brasilia: Autor.

. (1998). Cincuenta años de pensa $\neg$ miento de la Cepal: textos seleccionados. Santiago de Chile: Fondo de Cultura Económica.

Conesa, E. (1982). Conceptos fundamentales de la integración económica. Estudios Económicos. Revista de la Intal, 2-27. Recuperado de http://www10.iadb.org/intal/intalcdi/integracion_latinoamericana/documentos/071-Estudios_Economicos.pdf

Devlin, R. \& Estevadeordal, A. (2001). What's new in the new regionalism in the Americas? Working Paper $n .{ }^{\circ}$ 6. Washington: Inter-American Development Bank. Recuperado de http://www6.iadb.org/res/publications/pubfiles/pubS-106.pdf

Dollar, D. \& Kraay, A. (2001). Comercio exterior, crecimiento y pobreza. Finanzas y Desarrollo, 38(3), 16-19.

Echavarría, J. \& Villamizar, M. (eds.). (2006). Borradores de Economía n. ${ }^{\circ} 361$. El proceso colombiano de desindustrialización. Bogotá: Banco de la República.

Eduards, S. (1998). Openness, productivity and growth: What do we really know? The Economic Fournal, 108(447), pp. 383-398. Recuperado el 14 de octubre de 2008, de http://www.jstor.org/stable/2565567

Ffrench-Davis, R., Muñoz, O. \& Palma, G. (1998). The Latin American economies, 1959-1990. En: Bethell, L. (comp.). Latin America: Economy and society since 1930. Cambridge: Cambridge University Press.

Frenkel, R. \& Rapetti, M. (2011). Fragilidad externa o desindustrialización: ¿cuál es la principal amenaza para América Latina en la próxima década? Santiago de Chile: ONU. 
ISSN: 0124-3551 / Año 16, No 24 / enero-junio / pp. 195-215

Recuperado el 12 de noviembre de 2013, de http://www.cepal.org/de/publicaciones/xml/2/45172/Serie_MD_116.pdf

Garay, L. J. (2002). Colombia: entre la exclusión social y el desarrollo. Propuestas para la transición al Estado Social de Derecho. Bogotá: Contraloría General de la República.

Garay, L. J. \& Estevadeordal, A. (1996). Protección, desgravación preferencial y normas de origen en las Américas. En: Krugman, P., et. al. (1996). Las Américas. Integración económica en perspectiva. Bogotá: DPN/BID.

Gómez, I., Moreira, R. \& Santamaría, M. (1994). Los viejos criterios del nuevo arancel. Revista de Planeación y Desarrollo, 25(1), 231-265.

Gómez, I. (2013). El actual viejo debate de apertura y crecimiento. Suma de Negocios, 4(2), 81-95.

Grossman, G. \& Helpman, E. (1991). Innovation and growth in the global economy. Cambridge: MIT Press

Guerra-Borges, A. (2004). América Latina: regionalizar la globalización. De la trayectoria histórica a la utopía convocante. Economía UNAM, 1(002), 110-126. Recuperado el 10 de julio de 2014, de http://www.revistas.unam.mx/index.php/ecu/article/ view/2807/2367

Guillén-Romo, H. (2001). De la integración cepalina a la neoliberal en América Latina: de la ALALC al TLCAN. Comercio Exterior, 51(5).

Guillén-Romo, H. (2013). México: de la sustitución de importaciones al nuevo modelo económico. Comercio Exterior, 63(4), 34-60. Recuperado el 11 de julio de 2004, de http://revistas.bancomext.gob.mx/rce/magazines/157/6/Mexico-de_la_sustitucion.pdf

Hamilton, A. (1934). Secretary of the Treasury. Report on manufactures. Communicated to the House of Representatives, 5 de diciembre de 1791. En: McKee, S. Jr. (ed.). Papers on public credit, commerce and finance. Nueva York: Columbia University Press.

Hirschman, A. O. (1958). The strategy of economic development. New Haven: Yale University Press.

. (1971). The political economy of import-substituting industrialization in Latin America. En: A bias for hope: Essays on development and Latin America. New Haven: Yale University Press. 
Kay, C. (1998). Estructuralismo y teoría de la dependencia en el periodo neoliberal. Una perspectiva latinoamericana. Nueva Sociedad, (158), 100-119.

Krugman, P. (1994). The myth of Asia's miracle. Foreign Affairs, 73(6), 62-78.

(1996). Acuerdos comerciales e integración regional. En: Las Américas: integración económica en perspectiva. Bogotá: DNP/BID.

Krugman, P. \& Obstfeld M. (2001). Economía internacional, teoría y política (5.a ed.). Bogotá: Editorial Pearson.

Leamer, E. (1988). Measures of openness. En: Baldwin, R. (ed.). Trade policy and empirical analysis. Chicago: University of Chicago Press.

Levine, R. \& Renelt, D. (1992). A sensitivity analysis of cross-country growth regressions. American Economic Review, 82(4), 942-963.

List, F. (1856). National system or political economy. Filadelfia: J.B. Lippincott \& Co.

Love, J. (1994). Economic ideas and ideologies in Latin America since 1930. En: Bethel, L. (comp.). The Cambridge history of Latin America. Cambridge: Cambridge University Press.

Lucas, R. E. (1988). On the mechanics of economic development. Fournal of Monetary Economics, 22(1), 3-42.

McMillan, M., Horn, K. \& Rodrik, D. (2004). When economic reform goes wrong: Cashews in Mozambique. Working Paper n. ${ }^{\circ}$ 9117. Cambridge: National Bureau of Economic Research - NBER. Recuperado de http://www.nber.org/papers/w9117.pdf

Mill, J. S. (1987). Principles of political economy. Fairfield: Augustus M. Kelley Publishers. . (1861). El utilitarismo. Barcelona: Ediciones Altaya. . (1997). Sobre la libertad. Bogotá: Alianza Editorial.

Misas, G. (2002). La ruptura de los 90: del gradualismo al colapso. Bogotá: Universidad Nacional de Colombia.

Morales-Fajardo, M. E. (2007). Un repaso a la regionalización y el regionalismo: los primeros procesos de integración regional en América Latina. Confines de Relaciones Internacionales y Ciencia Política, 3(6), 65-80. Recuperado el 12 de julio de 2014, de http://www.redalyc.org/pdf/633/63300606.pdf 
ISSN: 0124-3551 / Año 16, No 24 / enero-junio / pp. 195-215

Obstfeld, M., \& Rogoff, K. (2000). New directions for stochastic open economy models. Journal of international economics, 50(1), 117-153.

Ocampo, J. A. \& Martínez, A. (2011). Política industrial de nueva generación para Colombia. Bogotá: Coalición para la Promoción de la Industria Colombiana.

Ocampo, J. A. (2001). El proceso de inserción internacional de América Latina y el Caribe. Documento presentado en el foro "La Internacionalización de Empresas Colombianas", Universidad de los Andes, Bogotá, Colombia.

. (2004). La América Latina y la economía mundial en el largo siglo XX. El Trimestre Económico, 71(4).

- (2008). Los paradigmas del desarrollo en la historia latinoamericana. En: Machinea, J. L., Iglesias, E. y Altimir, Ó. (eds.). Hacia la renovación de los paradigmas del desarrollo en América Latina. Santiago de Chile: Cepal/SEGIB.

Peñaranda, C. (1984). Estrategia para la integración económica en América Latina. Revista de la Intal, (3), p. 22-32. Recuperado el 28 de julio de 2014, de: http://www10. iadb.org/intal/intalcdi/integracion_latinoamericana/documentos/088-Estudios_ Economicos_2.pdf

Pipitone, U. (1996). Los nuevos sujetos regionales: raíces históricas y reordenamiento mundial. En: Borja, A., et al. Regionalismo y poder en América: los límites del neorrealismo. México D.F.: CIDE/Editorial Porrúa.

Pulecio, J. R. (2002). Estudios sobre el ALCA. El ALCA en la perspectiva de Colombia. Santiago de Chile: Fundación Friedrich-Ebert-Stiftung. Recuperado el 12 de julio de 2014, de http://library.fes.de/pdf-files/bueros/chile/01566.pdf

Quitral, M. (2009). La integración económica latinoamericana en tiempos de crisis: alcances y limitaciones para su consolidación. Nueva Sociedad, (222), 30-40. Recuperado el 28 de julio de 2014, de http://www.nuso.org/upload/articulos/3619_1.pdf

Ramonet, I. (2000). Economía política de la globalización. Barcelona: Editorial Ariel.

Reinert, E. S. (2007). How rich countries got rich and why poor countries stay poor. Nueva York: Carroll \& Graf Publishers.

Riveiro, D. (2005). Efectos potenciales de un proceso de integración. Revista Galega de Economía, 14(1-2), 1-34. Recuperado el 25 de julio de 2014, de http://www.usc.es/ econo/RGE/Vol14_1_2/Castelan/art18c.pdf 
Robinson, J. (1946). The pure theory of international trade. Review of Economic Studies, 14, 98-112.

Rodrik, D. (1995). Trade policy and industrial policy reform. En: Behrman, J. \& Srinivasan, T. N. (eds.). Handbook of development economics. Amsterdam: North Holland. . (2007). One economics, many recipes. Princeton: Princeton University Press.

Rodrik, D. (ed.). (2003). In search of prosperity: Analytic narratives on economic growth. Princeton: Princeton University Press.

Rodrik, D. \& Rosenzweig, M. R. (2009). Development policy and development economics: An introduction. En: Behrman, J. \& Srinivasan, T. N. (eds.). Handbook of development economics. Ámsterdam: Elsevier Science Publisher.

Rodrik, D. (2011). La paradoja de la globalización. Barcelona: Antoni Bosch Editor.

Romer, P. M. (1986). Increasing returns and long run growth. Fournal of Political Economy, 94(5), 1002-1037.

- (1992). Two strategies for economic development: Using ideas and producing ideas. Documento presentado en la Conferencia Anual del Banco Mundial sobre Desarrollo Económico, Banco Mundial, Washington, Estados Unidos.

Sachs, J. \& Warner, A. (1995). Economic reform and the process of global integration. Brooking Papers on Economic Activity, 1, 1-118.

Salama, P. (2012). Globalización comercial: desindustrialización prematura en América Latina e industrialización en Asia. Comercio Exterior, 62(6), 34-44.

Simonato R. (1985). El argumento de la industria incipiente y las políticas de industrialización selectivas. Económica, 31(1), 99-127.

Stiglitz, J. (2002). El malestar en la globalización. Madrid: Editorial Taurus.

Tamames, R. (2010). Estructura económica internacional. Madrid: Editorial Ariel.

Tinbergen, J. (1954). Integración económica internacional. Michigan: Elsevier University.

Winters, A.; Mc Culloch, N., and Mckay, A. (2004) Trade Liberalization and Poverty: The Evidence So Far. Journal of Economic Literature Vol. XLII (March 2004) pp. $72-115$. 